\title{
Self-Organizing Algorithm for Massive Tractography Datasets Clustering with Outliers Elimination Based on Multiple Species Flocking Model
}

Type of article: Conference abstract

Amira Chekir

LRPE Laboratory, FEI, USTHB University, Algiers, Algeria Corresponding Author: achekir@usthb.dz

\begin{abstract}
:
Background: The study of White Matter (WM) connectivity is of general interest in neuroscience, which is achieved by the analysis and clustering of the streamlines composed of the tractography dataset. The WM streamlines clustering is a challenge, because of the complexity and the vast size of the WM tractography dataset and its composition by various streamlines, in additionally to the presence of outliers.

Methods: Several WM clustering methods have been proposed in the literature to overcome these constraints. However, these methods stay statics. Once the clustering streamline is performed, it stays in this one. In this paper, we propose a new framework of distributed multiagent, improving, and adapting a bio-inspired model called Multiple Species Flocking (MSF) for WM streamlines clustering and automatic outlier elimination.

Results: The basic MSF rules are modified and adapted to perform streamlines clustering in higher dimensions. Specifically, each streamline is associated with a mobile agent and move onto a virtual space to form a group following the defined rules. Only the agents assigned to similar streamlines form a flock, whereas the agents assigned to dissimilar streamlines are sidelined and considered as outliers.

Conclusion: Swarm intelligence features of the approach, such as adaptivity, parallelism, dynamism, and decentralization, make our algorithm scalable to large datasets, very fast and accurate, which are confirmed by experimental results on synthetic and real datasets.

Keywords: White Matter, Clustering, Outliers Detection, Multiple Species Flocking Model, Swarm Intelligence, Multi-Agent Systems.
\end{abstract}

\section{Conflict of interest statement}

This article is a conference abstract presented at the International Congress on Health Sciences and Medical Technologies, Tlemcen Algeria 5-7 December 2019, ICHSMT'19.

\section{Authors' biography}

No Biography.

\section{References}

No references 